\title{
Constant Darkness Is a Mammalian Biological Signal
}

\author{
C.C. LEE \\ Department of Biochemistry and Molecular Biology, University of Texas \\ Health Science Center, Houston, Texas 77030
}

\begin{abstract}
Environmental light is a potent modulator of mammalian circadian rhythm and expression of clock genes. Constant darkness (DD) is regarded as a "free-running" circadian state. In nature, hibernating mammals encounter constant darkness (DD) seasonally. Circadian expression of enzymes involved in fat catabolism, procolipase (CLP) and pancreatic-lipase-related protein 2 (PLRP2), were identified in many peripheral organs of mice during DD but not during regular light/dark (LD) cycles. Circulating 5'-adenosine monophosphate (5'-AMP) was associated with DD-activated gene expression. Synthetic 5'-AMP, when injected into LD mice, activated procolipase expression in their peripheral organs and the animals become severely hypothermic, both key features of hibernating mammals. These findings identified a circadian-regulated metabolic cycle in mammals that may be associated with hypometabolic behaviors such as hibernation and torpor.
\end{abstract}

\section{INTRODUCTION}

Hypometabolism of cells and organs as a way to reduce ischemic damage holds enormous clinical applications (Kabon et al. 2003). Natural hypometabolism can be observed in animals that are able to undertake torpor, hibernation, or estivation. These behaviors, which reflect the different degrees of hypometabolism in diverse animal species, are thought to have very similar physiological controls (Heldmaier et al. 2004). However, little is known about the biochemical and cellular mechanisms underlying these behaviors in mammals.

In mammals, association between daily torpor and the body temperature rhythms implicates the circadian clock in the temporal control of this behavior (Perret and Aujard 2001; Heller and Ruby 2004). The observation that the ablation of the suprachiasmatic nucleus (SCN), the central endogenous clock synchronizer, abolished the torpor rhythm further implicates the circadian clock in such behavior (Ruby et al. 2002). Diverse mammalian species, from bears to primates such as the Malagasy lemurs, are known to undertake severe hypometabolic states such as torpor and hibernation (Heldmaier et al. 2004). These observations suggest the underlying biochemical processes for hypometabolism could be preserved in many mammalian species including humans.

It is widely recognized that mammals seasonally encounter prolonged DD during hibernation (Ruby et al. 1996; Heldmaier et al. 2004). Although regarded as a "free-running" state, the possibility that DD is a biological signal in mammals has never been excluded. Hence, a project was initiated to probe into the possibility of identifying genes activated in one aspect of the environments encountered during hibernation such as DD.

\section{PERIPHERAL ORGAN GENES ACTIVATED BY DD ENVIRONMENT}

Using a mouse gene library, a microarray comparison of gene expression was undertaken using liver mRNA from an animal kept in 48-hour DD (12:12-hour dark:dark) and an animal kept in a regular LD 12:12-hour cycle. (Zhang et al. 2006). This microarray comparison identified a gene encoding procolipase that was highly expressed in the liver from the animal kept in DD. Previous studies have shown that expression of the procolipase gene is highly specific to the pancreas and gastrointestinal tract, consistent with its role in the catabolism of dietary fat into fatty acids (Lowe 1977). However, in hibernating ground squirrels, expression of procolipase mRNA was activated in other peripheral organs, suggesting that our findings could be linked to such hypometabolic behavior (Squire et al. 2003). Northern blot analysis confirmed that the procolipase gene was activated in a circadian manner in peripheral organs when mice were kept in DD but not during LD cycles (Fig. 1a). Time course studies of procolipase expression over 48 hours in various tissues demonstrated a highly synchronized circadian control (Fig. 1b). Colipase is necessary for the enzymatic activity of pan-

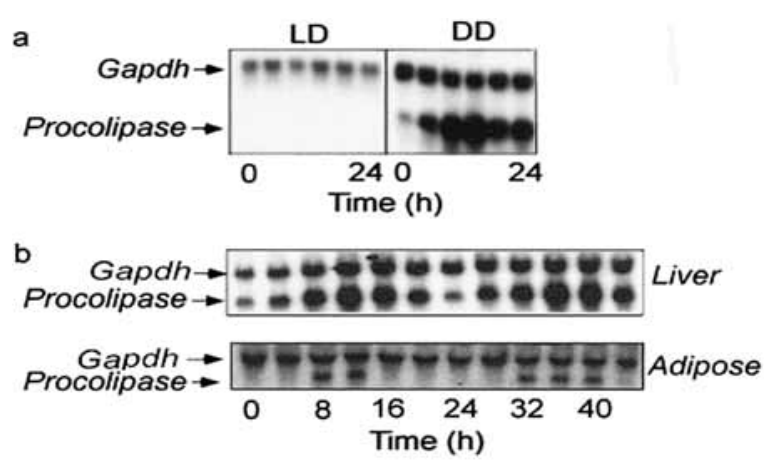

Figure 1. Activation of procolipase expression by DD. (a) Northern blot analysis showed the liver mRNA species of Gapdh-and procolipase-obtained mice kept in 12:12-hour LD or after 2 days in DD cycles or constant darkness environment. (b) A time course of procolipase expression in liver and adipose tissue during constant darkness. A housekeeping gene Gapdh was used as an internal control for relative mRNA levels. The respective genes were identified by radiolabeled cDNA probe for Gapdh and procoliapse, respectively. 
creatic lipases (Squire et al. 2003). Expression analysis of the gene encoding PLRP2 demonstrated that it was coordinately expressed with procolipase (Zhang et al. 2006). These observations indicated that DD-activated gene regulation is part of a complex biological program and is not unique to procolipase. Exposing mice kept in DD to white light resulted in the shut down of the procolipase expression in the various organs (Zhang et al. 2006). Together, these findings implicated a circulatory factor in the activation of procolipase expression during DD.

\section{IDENTIFICATION OF 5'-AMP AS A MEDIATOR OF PROCOLIPASE EXPRESSION}

On the basis of the above observations, the endogenous regulator was rationalized to have the following properties: (1) It is a circulatory molecule that must display circadian profile in its activity or levels and (2) it could act either as an activator or as a repressor. If it is an activator, then its injection into mice kept in the LD cycle will result in the induction of procolipase expression in the major organs. However, as a repressor, its injection into DD mice will abolish its procolipase expression in the peripheral organs.

Through a series of careful experiments, our search for this circulatory molecule was narrowed to the soluble nonpolypeptide aqueous fraction of blood extracts. Fractionation of the blood extracts by reverse-phase highperformance liquid chromatography (HPLC) revealed four highly reproducible peaks, of which peak 2 had a robust circadian pattern (Fig. 2). This peak also showed a strong diurnal profile with lower amplitude in LD mice (Zhang et al. 2006). Its characterization revealed a strong spectral absorption at $260 \mathrm{~nm}$, suggesting that this molecule was a nucleoside or nucleotide. Comparison of its fractionation distance on HPLC to chemical nucleotide standards demonstrated that it matches exactly to synthetic 5'-AMP (Fig. 3a). Identification of this molecule as 5 -AMP was confirmed by enzymatic analysis with snake venom nucleotidase, which dephosphorylated 5'-AMP into adenosine (Fig. 3b,c).

To demonstrate that $5^{\prime}$-AMP was the endogenous molecule associated with DD activation of procolipase expression, synthetic $5^{\prime}$-AMP was injected into LD mice. By reverse transcriptase-polymerase chain reaction (RTPCR), procolipase expression was detected in all tissues sampled with the exception of the brain from LD mice

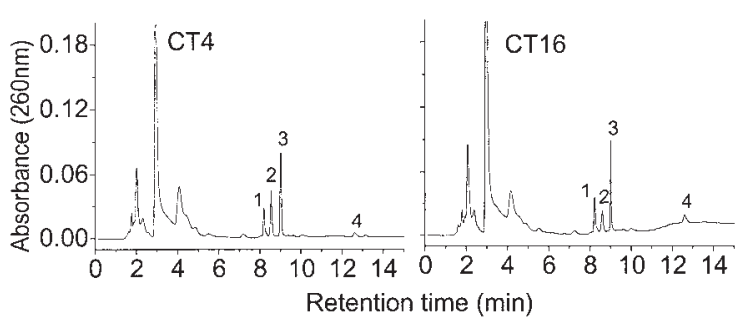

Figure 2. Identification of a circadian circulatory factor in blood. Extract was prepared from blood obtained from mice kept in 48-hour DD at CT4 and CT16. The aqueous phase was fractionated by HPLC, revealing four highly reproducible peaks (1, 2,3 , and 4 ). Note the robust circadian profile of peak 2 .

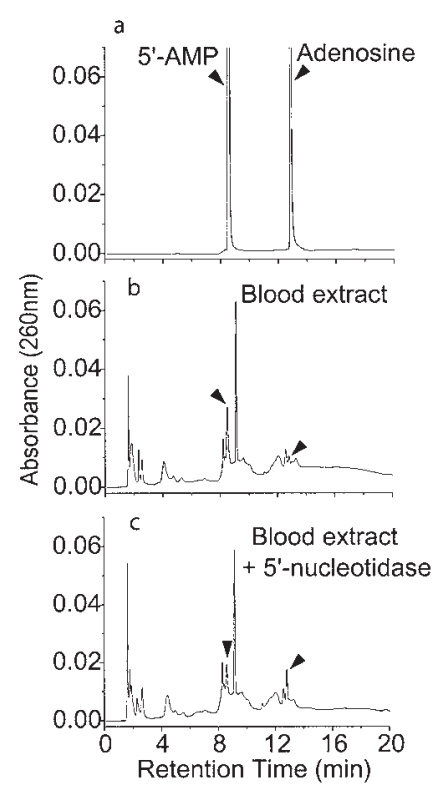

Figure 3. Identification of $5^{\prime}$-AMP as the circulatory factor. (a) Separation of chemical standards of $5^{\prime}$-AMP and adenosine under similar HPLC conditions. (b) Fractionation of blood extract; $(c)$ fractionation of the same blood extract after treatment by snake vernom nucleotidase. Note the reciprocal change in peak sizes of 5'-AMP with adenosine.

given 5'-AMP (Fig. 4a). In contrast, saline-injected LD animals display expression of procolipase only in the pancreas and the stomach, consistent with previous observations (Lowe 1977; Squire et al. 2003). These findings demonstrate that the elevated circulating level of 5 '-AMP was indeed associated with procolipase expression.

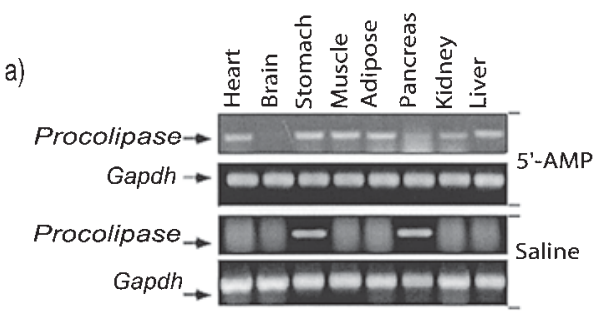

b)

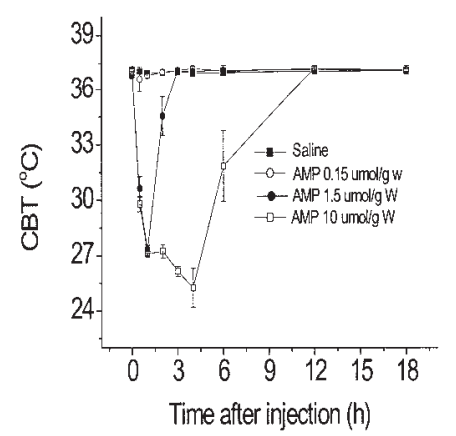

Figure 4. Induction of procolipase expression and hypothermia by synthetic $5^{\prime}$-AMP. (a) RT-PCR detection of procolipase and Gapdh expression in mice kept in 12:12-hour LD cycles given saline or 5 'AMP. (b) Hypothermic response of mice injected with various dosages of $5^{\prime}$-AMP. 


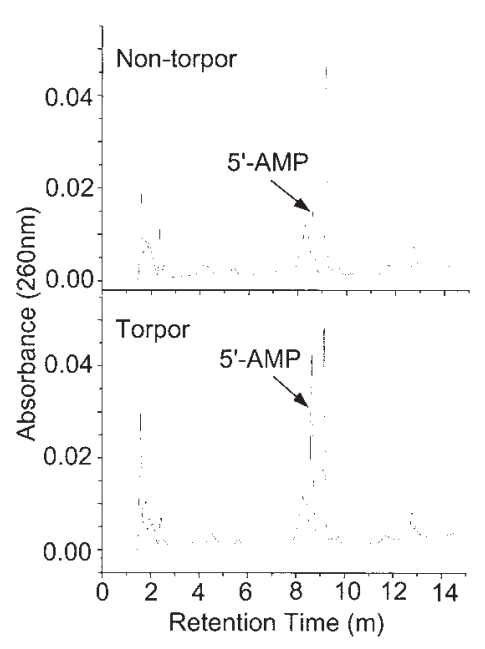

Figure 5. Rise in blood 5'-AMP levels during fasting-induced torpor. (a) HPLC fractionation of blood extract obtained from DD mice that were fed and not in torpor. (b) HPLC fractionation of blood extract obtained from a DD mouse that was in torpor induced by fasting.

The induction of procolipase by $5^{\prime}$-AMP has a prolonged time course, suggesting that its role in gene activation was likely indirect in vivo. Unexpectedly, mice that were given $5^{\prime}$-AMP were also severely hypothermic, with core body temperature as low as $25^{\circ} \mathrm{C}$ when kept in ambient room temperature (AET) of about $24^{\circ} \mathrm{C}$ (Fig. 4b). This hypothermic state was transient, and core body temperature was restored several hours later with no apparent shortcomings. These observations raised the possibility that 5'-AMP may have a similar role in natural torpor behavior.

This possibility was addressed by fasting mice kept in DD to induce natural torpor. HPLC analysis revealed that mice in torpor induced by fasting have a highly elevated circulatory $5^{\prime}$-AMP level. In contrast, mice in a similar environment that did not enter torpor and were fed have much lower circulatory 5'-AMP (Fig. 5). Whereas the kinetics of natural torpor was slower than that induced by synthetic 5'-AMP, this can be accounted for by the slower build up of natural $5^{\prime}$-AMP generated by fasting and its impact on thermal regulatory inhibition. Together, these observations revealed that an increase in natural 5 -AMP was associated with fasting-induced torpor.

\section{CONSTANT DARKNESS AND ENDOGENOUS METABOLIC RHYTHM}

Cessation of food intake and the generation of endogenous energy from fat are some of the physiological hallmarks of an animal in a deep hypometabolic state such as hibernation. The targeted activation of procolipase by DD must be physiological because procolipase mRNA encodes two peptides that are important for these hypometabolic events. The amino-terminal sequence of procolipase is a pentapeptide (VPDPR) that is posttranslationally cleaved from the colipase enzyme. This VPDPR peptide, named enterostatin, has been shown to act as a satiety inhibitor
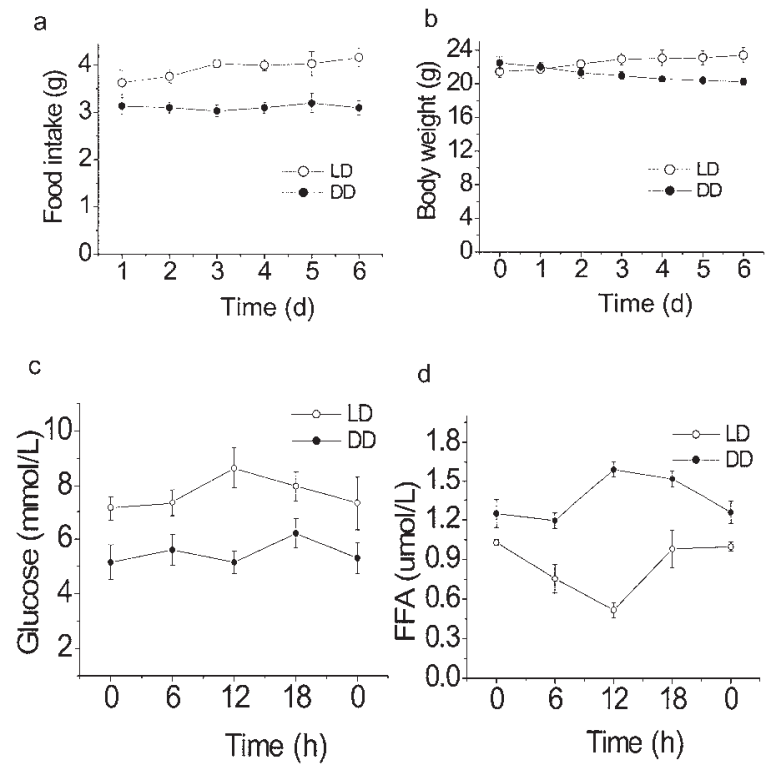

Figure 6. Effects of DD on metabolic status. (a) Daily food intake of mice in LD and DD cycles; $(b)$ body weight of mice during LD and DD cycles; (c) temporal rhythm of blood glucose during LD and DD cycles; $(d)$ temporal blood fatty acids level during LD and DD cycles. Note that all measurements were performed after DD mice had been in DD for 48 hours.

(Erlanson-Albertsson and Larsson 1988). A predicted outcome of the activated procolipase expression during the DD cycle would be an increase in VPDPR peptide production, which in turn should decrease satiety. Our studies revealed that DD mice consumed less food than LD mice during a 7-day study period (Fig. 6a). These observations were consistent with previous observations of rats displaying lower satiety in DD compared to those maintained in LD cycles (Stoynev and Ikonomov 1983). Consistent with the decrease in solid food intake was the observation that the body weight of the DD animals was moderately lower than that of mice kept in LD cycles over the same corresponding period (Fig. 6b). The broad activation of procolipase expression during DD cycles suggested that fat catabolism must be activated in these peripheral organs. A predicted outcome of such an increase in fat catabolism would be elevated serum free fatty acids. Indeed, DD mice have higher levels of free fatty acids in their serum compared to LD animals (Fig. $6 d)$. These findings are consistent with previous observations that large mammals kept in DD have higher serumfree fatty acids than those maintained in LD environment (Alila-Johansson et al. 2004). The observed increase in the use of fatty acids as fuel is complemented by a decline in blood glucose levels of DD animals compared to LD mice (Fig. 6c). This difference in preference of fatty acids over glucose as fuel in DD mice is reminiscent of animals in hibernation.

Membrane-anchored and circadian-regulated ecto5 'nucleotidase controls the circulatory 5'-AMP levels and mediates its intracellular action (von Mayersbach and Klaushofer 1979; Uchiyama 1983; Thompson et al. 1989). The extracellular ecto-5'nucleotidase dephospho- 


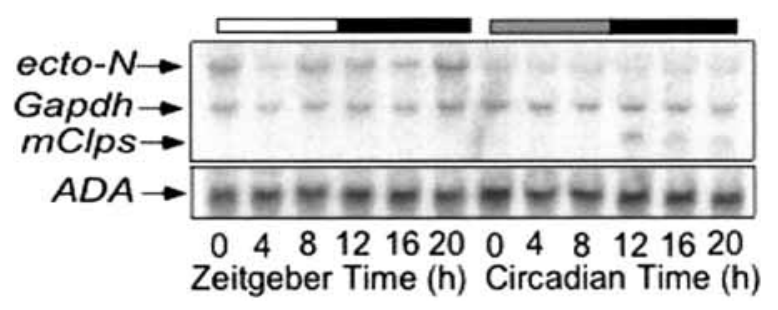

Figure 7. Inhibition of ecto-5'nucleotidase mRNA expression by DD. Shown here is northern blot analysis for expression of ecto-5' nucleotidase (ecto- $N$ ), Gapdh, procolipase ( $m$ Clps), and adenosine deaminase $(A D A)$ in mice liver mRNA obtained every 4 hours from animals kept in LD or DD cycles. The mRNA expression levels were identified by radiolabeled cDNA probe for the respective genes.

rylates $5^{\prime}$-AMP to adenosine, which is taken rapidly into the cell by nucleoside transporters (Thorn and Jarvis 1996). Intracellular adenosine is primarily phosphorylated to $5^{\prime}$-AMP by adenosine kinase because its $K_{\mathrm{m}}$ for adenosine is one or two orders of magnitude lower than that of adenosine deaminase (ADA) (Schnebli et al. 1967; Arch and Newsholme 1978). Northern blot analysis demonstrates that the expression of the ecto-5'nucleotidase mRNA in LD mice liver was regulated in a circadian manner and was significantly dampened in DD animals (Fig. 7). In contrast, $A D A$ mRNA expression was unaffected by changes in lighting regime. The repression of ecto- $5^{\prime}$ nucleotidase expression by DD is opposite to the activation observed for procolipase and its enzymatic partners. These observations demonstrate that DD regulation of gene expression was not narrowly restricted to those of procolipase and its enzymatic partners but is coordinated to involve regulators of circulatory adenylate levels.

\section{DISCUSSION}

Although ATP is the cellular energy currency, 5'-AMP occupies the unique biochemical position that determines salvage or catabolism of cellular adenine nucleotides (Lehninger 1977). The adenylate biochemical equilibrium, ATP $+5^{\prime}$-AMP $\leftrightarrow 2 \mathrm{ADP}$, which is regulated by the enzyme adenylate kinase, controls cellular energy charge. In addition, $5^{\prime}$-AMP can act as either a positive or a negative allosteric regulator of enzymes that controls glucose homeostasis such as fructose 1,6 phosphatase $(F D P)$ and phosphofructose kinase $(P F K)$, respectively. $F D P$ is a rate-limiting enzyme for gluconeogenesis and it converts fructose 1,6 phosphates to fructose 6-phosphate. FDP binding of 5'-AMP will inhibit its enzymatic activity, thereby limiting gluconeogenesis. On the reverse direction, $P F K$ is a rate-limiting enzyme for glycolysis. $P F K$ converts fructose 6-phosphate into fructose 1,6 phosphate utilizing an ATP molecule. Unlike FDP, the activity of $P F K$ is enhanced by $5^{\prime}$-AMP thereby increasing the rate of glycolysis. For both enzymes, ATP has the opposite allosteric effects to 5'-AMP because these adenylate nucleotides bind competitively to the same regulatory motif (Kemp and Gunasekera 2002). Therefore, a change in the ATP to 5'-AMP ratio will regulate the cellular energy demand and is directly linked to biochemical processes that regulate glucose production and its utilization. In turn, gluconeogenesis performed primarily in the liver is largely dependent on ATP and NADH generated by fat catabolism. Our observations indicate that the activation of procolipase expression is linked to a change in amplitude of blood glucose levels (Zhang et al. 2006). The current observations raise the question of why the animal maintains such a complex cellular mechanism to maintain circadian rhythm for circulating $5^{\prime}$-AMP. Could it be that the temporal changes in circulating $5^{\prime}$-AMP levels was reflecting the state of the energy demands in vivo? That is, 5 -AMP is a necessary pivotal metabolic signal for the organism to maintain metabolic homeostasis.

In conclusion, our studies demonstrated that induction of procolipase expression by a DD cycle via $5^{\prime}$-AMP is part of a complex biochemical and physiological process that accompanies a change in environmental conditions.

\section{ACKNOWLEDGMENTS}

This work is supported in part by a National Institutes of Health Director Pioneer Award to the author. The author is indebted to Drs. J. Zhang and K. Kassik for their contributions to this chapter.

\section{REFERENCES}

Alila-Johansson A., Eriksson L., Soveri T., and Laakso M.L. 2004. Daily and annual variations of free fatty acid, glycerol and leptin plasma concentrations in goats (Capra hircus) under different photoperiods. Comp. Biochem. Physiol. A Mol. Integr. Physiol. 138: 119.

Arch J.R. and Newsholme E.A. 1978. Activities and some properties of 5 '-nucleotidase, adenosine kinase and adenosine deaminase in tissues from vertebrates and invertebrates in relation to the control of the concentration and the physiological role of adenosine. Biochem. J. 174: 965.

Erlanson-Albertsson C. and Larsson A. 1988. The activation peptide of pancreatic procolipase decreases food intake in rats. Regul. Pept. 22: 325.

Heldmaier G., Ortmann S., and Elvert R. 2004. Natural hypometabolism during hibernation and daily torpor in mammals. Respir Physiol. Neurobiol. 141: 317.

Heller H.C and Ruby N.F. 2004. Sleep and circadian rhythms in mammalian torpor. Annu. Rev. Physiol. 66: 275.

Kabon B., Bacher A., and Spiss C.K. 2003. Therapeutic hypothermia. Best Pract. Res. Clin. Anaesthesiol. 17: 551.

Kemp R.G. and Gunasekera D. 2002. Evolution of the allosteric ligand sites of mammalian phosphofructo-1-kinase. Biochemistry 41: 9426.

Lehninger A.L. 1977. Biochemistry: The molecular basis of cell structure and function, 2nd edition, p 623. Worth, New York.

Lowe M.E. 1977. Molecular mechanisms of rat and human pancreatic triglyceride lipases. J. Nutr. 127: 549.

Perret M. and Aujard F. 2001. Daily hypothermia and torpor in a tropical primate: Synchronization by $24-\mathrm{h}$ light-dark cycle. Am. J. Physiol. Regul. Integr. Comp. Physiol. 281: R1925.

Ruby N.F., Dark J., Heller H.C., and Zucker I. 1996. Ablation of suprachiasmatic nucleus alters timing of hibernation in ground squirrels. Proc. Natl. Acad. Sci. 93: 9864.

Ruby N.F., Dark J., Burns D.E., Heller H.C., and Zucker I. 2002. The suprachiasmatic nucleus is essential for circadian body temperature rhythms in hibernating ground squirrels. $J$. Neurosci. 22: 357.

Schnebli H.P., Hill D.L., and Bennett L.L., Jr. 1967. Purification and properties of adenosine kinase from human tumor cells of type H. Ep. No. 2. J. Biol. Chem. 242: 1997. 
Squire T.L., Lowe M.E., Bauer V.W., and Andrews M.T. 2003. Pancreatic triacylglycerol lipase in a hibernating mammal. II. Cold-adapted function and differential expression. Physiol. Genomics 16: 131.

Stoynev A.G. and Ikonomov O.C. 1983. Effect of constant light and darkness on the circadian rhythms in rats. I. Food and water intake, urine output and electrolyte excretion. Acta Physiol. Pharmacol. Bulg. 9: 58.

Thompson L.F., Ruedi J.M., Glass A., Low M.G., and Lucas A.H. 1989. Antibodies to $5^{\prime}$-nucleotidase (CD73), a glycosylphosphatidylinositol-anchored protein, cause human peripheral blood T cells to proliferate. J. Immunol. 143: 1815 .
Thorn J.A. and Jarvis S.M. 1996. Adenosine transporters. Gen. Pharmacol. 27: 613.

Uchiyama Y. 1983. A histochemical study of variations in the localization of $5^{\prime}$-nucleotidase activity in the acinar cell of the rat exocrine pancreas over the twenty-four hour period. Cell Tissue Res. 230: 411.

von Mayersbach H. and Klaushofer K. 1979. Circadian variations of 5'-nucleotidase activity in rat liver. Cell. Mol. Biol. Incl. Cyto Enzymol. 24: 73.

Zhang J., Kaasik K., Blackburn M.R., and Lee C.C. 2006. Constant darkness is a circadian metabolic signal in mammals. Nature 439: 340. 


\section{$8_{\text {SYMPOS }}^{\infty}$ Cold Spring Harbor Symposia SYMPOSIA on Quantitative Biology}

\section{Constant Darkness Is a Mammalian Biological Signal}

C. C. Lee

Cold Spring Harb Symp Quant Biol 2007 72: 287-291

Access the most recent version at doi:10.1101/sqb.2007.72.051

References This article cites 19 articles, 5 of which can be accessed free at:

http://symposium.cshlp.org/content/72/287.full.html\#ref-list-1

\section{License} Email Alerting $\begin{aligned} & \text { Receive free email alerts when new articles cite this article - sign up in the box at the } \\ & \text { Service }\end{aligned}$ top right corner of the article or click here. 